Article

\title{
Fibonacci Numbers with a Prescribed Block of Digits
}

\author{
Pavel Trojovský(i) \\ Department of Mathematics, Faculty of Science, University of Hradec Králové, 50003 Hradec Králové, \\ Czech Republic; pavel.trojovsky@uhk.cz
}

Received: 27 March 2020; Accepted: 19 April 2020; Published: 21 April 2020

\begin{abstract}
In this paper, we prove that $F_{22}=17711$ is the largest Fibonacci number whose decimal expansion is of the form $a b \ldots b c \ldots c$. The proof uses lower bounds for linear forms in three logarithms of algebraic numbers and some tools from Diophantine approximation.
\end{abstract}

Keywords: Fibonacci numbers; digits; linear forms in logarithms; Baker-Davenport

MSC: primary 11A63, 11B39; secondary 11J86

\section{Introduction}

Let $\left(F_{n}\right)_{n \geq 0}$ be the Fibonacci sequence given by second-order recurrence $F_{n+2}=F_{n+1}+F_{n}$, for $n \geq 0$, with initial conditions $F_{0}=0$ and $F_{1}=1$. A few terms of this sequence are

$0,1,1,2,3,5,8,13,21,34,55,89,144,233,377,610,987,1597,2584,4181,6765,10946,17711, \ldots$

In the last decades, many results on Diophantine properties of Fibonacci numbers have been proved with the use of refined tools in number theory. For instance, Bugeaud, Mignotte, and Siksek [1] settled the problem of Fibonacci perfect power numbers (i.e., the equation $F_{n}=y^{t}$, for $t>1$ ) by combing the powerful Baker's theory with the Modular method (used by Wiles in the proof of the Fermat Last Theorem). See a generalization of their result in [2].

We remark that digital problems involving Fibonacci numbers have received much attention in the literature. A first result in this direction was proved, in 2000, by F. Luca [3] who showed that the largest Fibonacci number with only one distinct digit is $F_{10}=55$. After this, many authors worked on repdigits (i.e., integers having only one distinct digit in its decimal expansion) as expressions related to sum, product of terms of binary recurrences (see [4-13] and references therein). However, the related problem of finding all Fibonacci numbers with only two distinct digits remains open.

The aim of this paper is to continue this program. In fact, our main result searches for all Fibonacci numbers of the form $a b \ldots b c \ldots c$, which provides a generalization for Luca's result $(a=b=c)$. More precisely, our main result is the following:

Theorem 1. Let $a \in[1,9]$ and $b, c \in[0,9]$. The largest solution of the Diophantine equation

$$
F_{n}=a \underbrace{b \ldots b}_{m} \underbrace{c \ldots c}_{\ell}=a \cdot 10^{m+\ell}+b \cdot\left(\frac{10^{m}-1}{9}\right) 10^{\ell}+c \cdot\left(\frac{10^{\ell}-1}{9}\right),
$$

in positive integers $m, n$, and $\ell$, with $2 \leq \ell \leq m$, is $n=22$. Explicitly, $F_{22}=17711$.

Our proof combines two deep techniques in number theory, namely, the Baker's theory on linear forms in logarithms and some tools from Diophantine approximation (a reduction method due to Baker and Davenport). 


\section{Auxiliary Results}

In this section, we shall present some results which will be useful in the proofs.

Let $\alpha=(1+\sqrt{5}) / 2$ and $\beta=-1 / \alpha$. The Binet's formula asserts that $F_{n}=\left(\alpha^{n}-\beta^{n}\right) / \sqrt{5}$. From this formula, it is possible to deduce that the estimates

$$
\alpha^{n-2} \leq F_{n} \leq \alpha^{n-1}
$$

hold for all $n \geq 1$. In addition, this Binet's formula allows us to manipulate our Diophantine equation to obtain upper bounds for some linear forms in three logarithms. Thus, in order to obtain lower bounds for these forms, we shall use the celebrated Baker's theory. Among these lower bounds, we decided to use one which was proved in ([1], Theorem 9.4).

Lemma 1. Let $\gamma_{1}, \ldots, \gamma_{t}$ be real algebraic numbers and let $b_{1}, \ldots, b_{t}$ be nonzero rational integer numbers. Let $D$ be the degree of the number field $\mathbb{Q}\left(\gamma_{1}, \ldots, \gamma_{t}\right)$ over $\mathbb{Q}$ and let $A_{j}$ be a positive real number satisfying

$$
A_{j} \geq \max \left\{D h\left(\gamma_{j}\right),\left|\log \gamma_{j}\right|, 0.16\right\}
$$

where $j=1, \ldots, t$. Assume that

$$
B \geq \max \left\{\left|b_{1}\right|, \ldots,\left|b_{t}\right|\right\}
$$

$$
\begin{aligned}
& \text { If } \gamma_{1}^{b_{1}} \cdots \gamma_{t}^{b_{t}} \neq 1 \text {, then } \\
& \quad\left|\gamma_{1}^{b_{1}} \cdots \gamma_{t}^{b_{t}}-1\right| \geq \exp \left(-1.4 \cdot 30^{t+3} \cdot t^{4.5} \cdot D^{2}(1+\log D)(1+\log B) A_{1} \cdots A_{t}\right) .
\end{aligned}
$$

Here, the logarithmic height of an $n$-degree algebraic number $\alpha$ is defined as

$$
h(\alpha)=\frac{1}{n}\left(\log |a|+\sum_{j=1}^{n} \log \max \left\{1,\left|\alpha^{(j)}\right|\right\}\right),
$$

where $a$ is the leading coefficient of the minimal primitive polynomial of $\alpha$ (over $\mathbb{Z}$ ) and $\left(\alpha^{(j)}\right)_{1 \leq j \leq n}$ are the (algebraic) conjugates of $\alpha$.

With these lower and upper bounds, we shall obtain an upper bound for $n$ which is, in general, very large and then the next step is to reduce it. For that, we shall use a reduction method which is originated from Diophantine approximation. Here, we shall use a result due to Dujella and Pethö [14] (which is a variant of a famous method due to Baker-Davenport). For a real number $x$ we use $\|x\|=\min \{|x-n|: n \in \mathbb{Z}\}$ for the distance from $x$ to the nearest integer (the so-called Nint function).

Lemma 2. Let $M>0$ be an integer and let $\gamma, \mu$ be real numbers, such that $\gamma \notin \mathbb{Q}$. Let $p / q$ be a convergent of the continued fraction expansion of $\gamma$ such that $q>6 M$ and $\epsilon:=\|\mu q\|-M\|\gamma q\|>0$. Then there is no solution to the Diophantine inequality

$$
0<m \gamma-n+\mu<A \cdot B^{-m}
$$

in positive integers $m, n$ with

$$
\frac{\log (A q / \epsilon)}{\log B} \leq m<M
$$

After presetting these tools, we can now prove our main result. 


\section{The Proof of The Theorem}

\subsection{Finding a Bound on $N$}

By the Binet's formula and the identity in Equation (1), we have

$$
\frac{\alpha^{n}}{\sqrt{5}}-\left(a+\frac{b}{9}\right) 10^{m+\ell}=\left(\frac{c-b}{9}\right) 10^{\ell}+\frac{\beta^{n}}{\sqrt{5}}-\frac{c}{9} .
$$

Thus,

$$
\left|\frac{\alpha^{n}}{\sqrt{5}}-\left(a+\frac{b}{9}\right) 10^{m+\ell}\right|<2 \cdot 10^{\ell} .
$$

On dividing through by $(a+b / 9) 10^{m+\ell}$, we obtain

$$
\left|\left(a+\frac{b}{9}\right)^{-1} \frac{\alpha^{n}}{\sqrt{5}} 10^{-(m+\ell)}-1\right|<\frac{2}{10^{m}}
$$

where we used the fact that $a+b / 9 \geq 1$. Now, we are in a position to apply Lemma 1 , but first we must prove that $\left|(\sqrt{5}(a+b / 9))^{-1} 10^{-(m+\ell)} \alpha^{n}-1\right| \neq 0$. Indeed, in the contrary case, we would get that $\alpha^{2 n} \in \mathbb{Q}$ which is an absurd. Thus, let us take

$$
\gamma_{1}:=\sqrt{5}(a+b / 9), \gamma_{2}:=\alpha, \gamma_{3}:=10, b_{1}:=-1, b_{2}:=n, b_{3}:=-(m+\ell) \text {. }
$$

Note that $\mathbb{Q}\left(\gamma_{1}, \gamma_{2}, \gamma_{3}\right)=\mathbb{Q}(\sqrt{5})$ and then $D=2$. The conjugates of $\gamma_{1}, \gamma_{2}$, and $\gamma_{3}$ are $\gamma_{1}^{\prime}=-\gamma_{1}, \gamma_{2}^{\prime}=\beta, \gamma_{3}^{\prime}=\gamma_{3}$, respectively. Surely, $\gamma_{2}$ and $\gamma_{3}$ are algebraic integers, while the minimal polynomial of $\gamma_{1}$ is $\left(X-\gamma_{1}\right)\left(X-\gamma_{1}^{\prime}\right)=X^{2}-5(a+b / 9)^{2}$ which is a divisor of $81 X^{2}-5(9 a+b)^{2}$. Therefore,

$$
h\left(\gamma_{1}\right) \leq \frac{1}{2}(\log 81+2 \log (10 \sqrt{5}))<5.4 .
$$

(A more relaxed upper bound for $h\left(\gamma_{1}\right)$ could be found by using the well-known property that $h(x+y z) \leq h(x)+h(y)+h(z)+\log 2)$. In addition, $h\left(\gamma_{2}\right)=(\log \alpha) / 2<0.75$ and $h\left(\gamma_{3}\right)=\log 10<$ 2.31. Let us take $A_{1}:=10.9, A_{2}=1.5$, and $A_{3}=4.7$. Of course, we can assume that $n>14$. Thus,

$$
\left|\left(a+\frac{b}{9}\right)^{-1} \frac{\alpha^{n}}{\sqrt{5}} 10^{-(m+\ell)}-1\right|>\exp \left(-7.6 \cdot 10^{13}(1+\log B)\right),
$$

where $B \geq \max \{n, m+\ell\}$

By combining the estimates in Equations (4) and (5), we get

$$
m<3.4 \cdot 10^{13}(1+\log B)
$$

Now, we have that $F_{n}$ has $m+\ell+1$ digits and so

$$
m+\ell+1=\left\lfloor\frac{\log F_{n}}{\log 10}\right\rfloor+1
$$

Since $m \geq \ell$ and $\lfloor x\rfloor+1>x$, we have

$$
2 m+1>\frac{\log F_{n}}{\log 10} \geq(n-2) \frac{\log \alpha}{\log 10}>0.2(n-2)
$$

so $10 m+6 \geq n$. Thus, we can take $B:=10 m+6$, which yields to 


$$
m<3.4 \cdot 10^{13}(1+\log (10 m+6))
$$

and therefore $m \leq 1.3 \cdot 10^{15}$.

\subsection{Reducing the Bound}

Now, let us write $\Lambda:=n \log \alpha-(m+\ell) \log 10+\log \left((a+b / 9)^{-1}(\sqrt{5})^{-1}\right)$. We know that $e^{x}-1>x$, for all $x \in \mathbb{R}^{+}$. By supposing that $\Lambda>0$ (the other case is completely similar, where we used the fact that $\left|e^{x}-1\right|=1-e^{-|x|}$, if $x<0$ ), we can rewrite Equation (4) as

$$
0<n \log \alpha-(m+\ell) \log 10+\log \left((a+b / 9)^{-1}(\sqrt{5})^{-1}\right)<1.8 \cdot 10^{-m} .
$$

Since $m>(0.1) n-0.7$ (because $2 m+1>0.2(n-2)$ ), we can divide the previous inequality by $\log 10$, to obtain

$$
0<n \gamma-(m+\ell)+\mu<4 \cdot(1.2)^{-n},
$$

where $\gamma:=\log \alpha / \log 10$ and $\mu:=\log \left((a+b / 9)^{-1}(\sqrt{5})^{-1}\right) / \log 10$.

Clearly $\gamma$ is an irrational number (because $\alpha^{k}$ is irrational for any non-zero integer $k$ ). Let us denote $p_{n} / q_{n}$ as the $n$th convergent of its continued fraction.

In order to reduce our bound on $m$, we shall use Lemma 2. Now, since $n \leq 10 m+6<1.4 \cdot 10^{16}$, we choose $M=1.4 \cdot 10^{16}$. Thus,

$$
\frac{p_{38}}{q_{38}}=\frac{1426134855866370784}{6824015306170795931}
$$

then $q_{38} \geq 6824015306170795931>8.4 \cdot 10^{16}=6 M$. Furthermore, we have $M\left\|q_{38} \gamma\right\|<0.0013$. On the other hand, by computing $\left\|q_{38} \mu\right\|$, for $a \in[1,9]$ and $b \in[0,9]$, we have that the minimal value of this expression is obtained when $a=8, b=2$ and is $>0.0028$. Hence,

$$
\epsilon=\left\|q_{38} \mu\right\|-M\left\|q_{38} \gamma\right\|>0.0015 .
$$

We notice the all the hypotheses of the Lemma 2 are fulfilled, where $A=4$ and $B=1.2$, so, by that lemma, there is no solution of the inequality in Equation (7) (and then for the Diophantine Equation (1)) for $n$ in the range

$$
\left[\left\lfloor\log \left(A q_{38} / \epsilon\right) / \log B\right\rfloor+1, M\right]=\left[281,1.4 \cdot 10^{16}\right] .
$$

Since $n<M$, we get $n \leq 280$. By using Equation (6), we deduce that

$$
m+\ell \leq(n-1) \frac{\log \alpha}{\log 10}-1<58.4
$$

and so $m+\ell \leq 58$. Since $m+\ell+1 \geq 5$, it is seen that $F_{n}$ has at least 5 digits yielding $n \geq 21$. A simple search in the list of the Fibonacci numbers $F_{n}$ in the range $n \in[21,280]$ (see Table A1 in Appendix A), returns only $F_{22}=17711$ with the required properties. This completes the proof.

\section{Conclusions}

In this paper we have been interested in finding all Fibonacci numbers which are special concatenation of digits. In particular, we show that $F_{22}=17711$ is the largest Fibonacci number whose decimal expansion is of the form $a b \ldots b c \ldots c$, where $a, b$, and $c$ are decimal digits. Our approach to the proof is based on the combination of lower bounds for linear forms in logarithms (due to Baker) with reduction methods (due to Dujella-Pethö).

Funding: The author was supported by Project of Excelence PrF UHK No. 2215/2020, University of Hradec Králové, Czech Republic. 
Acknowledgments: The author is very grateful to the referees for their very constructive suggestions that helped to improve the quality of this paper.

Conflicts of Interest: The author declares no conflict of interest.

\section{Appendix A}

Table A1. Values of $F(n)$ for $n$ from 21 to 280 .

\begin{tabular}{|c|c|c|c|}
\hline$n$ & $F_{n}$ & $n$ & $F_{n}$ \\
\hline 21 & 10946 & 280 & 14691098406862188148944207245954912110548093601382197697835 \\
\hline 22 & 17711 & 279 & 9079598147510263717870894449029933369491131786514446266146 \\
\hline 23 & 28657 & 278 & 5611500259351924431073312796924978741056961814867751431689 \\
\hline 24 & 46368 & 277 & 3468097888158339286797581652104954628434169971646694834457 \\
\hline 25 & 75025 & 276 & 2143402371193585144275731144820024112622791843221056597232 \\
\hline 26 & 121393 & 275 & 1324695516964754142521850507284930515811378128425638237225 \\
\hline 27 & 196418 & 274 & 818706854228831001753880637535093596811413714795418360007 \\
\hline 28 & 317811 & 273 & 505988662735923140767969869749836918999964413630219877218 \\
\hline 29 & 514229 & 272 & 312718191492907860985910767785256677811449301165198482789 \\
\hline 30 & 832040 & 271 & 193270471243015279782059101964580241188515112465021394429 \\
\hline 31 & 1346269 & 270 & 119447720249892581203851665820676436622934188700177088360 \\
\hline 32 & 2178309 & 269 & 73822750993122698578207436143903804565580923764844306069 \\
\hline 33 & 3524578 & 268 & 45624969256769882625644229676772632057353264935332782291 \\
\hline 34 & 5702887 & 267 & 28197781736352815952563206467131172508227658829511523778 \\
\hline 35 & 9227465 & 266 & 17427187520417066673081023209641459549125606105821258513 \\
\hline 36 & 14930352 & 265 & 10770594215935749279482183257489712959102052723690265265 \\
\hline 37 & 24157817 & 264 & 6656593304481317393598839952151746590023553382130993248 \\
\hline 38 & 39088169 & 263 & 4114000911454431885883343305337966369078499341559272017 \\
\hline 39 & 63245986 & 262 & 2542592393026885507715496646813780220945054040571721231 \\
\hline 40 & 102334155 & 261 & 1571408518427546378167846658524186148133445300987550786 \\
\hline 41 & 165580141 & 260 & 971183874599339129547649988289594072811608739584170445 \\
\hline 42 & 267914296 & 259 & 600224643828207248620196670234592075321836561403380341 \\
\hline 43 & 433494437 & 258 & 370959230771131880927453318055001997489772178180790104 \\
\hline 44 & 701408733 & 257 & 229265413057075367692743352179590077832064383222590237 \\
\hline 45 & 1134903170 & 256 & 141693817714056513234709965875411919657707794958199867 \\
\hline 46 & 1836311903 & 255 & 87571595343018854458033386304178158174356588264390370 \\
\hline 47 & 2971215073 & 254 & 54122222371037658776676579571233761483351206693809497 \\
\hline 48 & 4807526976 & 253 & 33449372971981195681356806732944396691005381570580873 \\
\hline 49 & 7778742049 & 252 & 20672849399056463095319772838289364792345825123228624 \\
\hline 50 & 12586269025 & 251 & 12776523572924732586037033894655031898659556447352249 \\
\hline 51 & 20365011074 & 250 & 7896325826131730509282738943634332893686268675876375 \\
\hline 52 & 32951280099 & 249 & 4880197746793002076754294951020699004973287771475874 \\
\hline 53 & 53316291173 & 248 & 3016128079338728432528443992613633888712980904400501 \\
\hline 54 & 86267571272 & 247 & 1864069667454273644225850958407065116260306867075373 \\
\hline 55 & 139583862445 & 246 & 1152058411884454788302593034206568772452674037325128 \\
\hline 56 & 225851433717 & 245 & 712011255569818855923257924200496343807632829750245 \\
\hline 57 & 365435296162 & 244 & 440047156314635932379335110006072428645041207574883 \\
\hline 58 & 591286729879 & 243 & 271964099255182923543922814194423915162591622175362 \\
\hline 59 & 956722026041 & 242 & 168083057059453008835412295811648513482449585399521 \\
\hline 60 & 1548008755920 & 241 & 103881042195729914708510518382775401680142036775841 \\
\hline 61 & 2504730781961 & 240 & 64202014863723094126901777428873111802307548623680 \\
\hline 62 & 4052739537881 & 239 & 39679027332006820581608740953902289877834488152161 \\
\hline 63 & 6557470319842 & 238 & 24522987531716273545293036474970821924473060471519 \\
\hline 64 & 10610209857723 & 237 & 15156039800290547036315704478931467953361427680642 \\
\hline 65 & 17167680177565 & 236 & 9366947731425726508977331996039353971111632790877 \\
\hline 66 & 27777890035288 & 235 & 5789092068864820527338372482892113982249794889765 \\
\hline 67 & 44945570212853 & 234 & 3577855662560905981638959513147239988861837901112 \\
\hline 68 & 72723460248141 & 233 & 2211236406303914545699412969744873993387956988653 \\
\hline 69 & 117669030460994 & 232 & 1366619256256991435939546543402365995473880912459 \\
\hline 70 & 190392490709135 & 231 & 844617150046923109759866426342507997914076076194 \\
\hline
\end{tabular}


Table A1. Cont.

\begin{tabular}{|c|c|c|c|}
\hline$n$ & $F_{n}$ & $n$ & $F_{n}$ \\
\hline 71 & 308061521170129 & 230 & 522002106210068326179680117059857997559804836265 \\
\hline 72 & 498454011879264 & 229 & 322615043836854783580186309282650000354271239929 \\
\hline 73 & 806515533049393 & 228 & 199387062373213542599493807777207997205533596336 \\
\hline 74 & 1304969544928657 & 227 & 123227981463641240980692501505442003148737643593 \\
\hline 75 & 2111485077978050 & 226 & 76159080909572301618801306271765994056795952743 \\
\hline 76 & 3416454622906707 & 225 & 47068900554068939361891195233676009091941690850 \\
\hline 77 & 5527939700884757 & 224 & 29090180355503362256910111038089984964854261893 \\
\hline 78 & 8944394323791464 & 223 & 17978720198565577104981084195586024127087428957 \\
\hline 79 & 14472334024676221 & 222 & 11111460156937785151929026842503960837766832936 \\
\hline 80 & 23416728348467685 & 221 & 6867260041627791953052057353082063289320596021 \\
\hline 81 & 37889062373143906 & 220 & 4244200115309993198876969489421897548446236915 \\
\hline 82 & 61305790721611591 & 219 & 2623059926317798754175087863660165740874359106 \\
\hline 83 & 99194853094755497 & 218 & 1621140188992194444701881625761731807571877809 \\
\hline 84 & 160500643816367088 & 217 & 1001919737325604309473206237898433933302481297 \\
\hline 85 & 259695496911122585 & 216 & 619220451666590135228675387863297874269396512 \\
\hline 86 & 420196140727489673 & 215 & 382699285659014174244530850035136059033084785 \\
\hline 87 & 679891637638612258 & 214 & 236521166007575960984144537828161815236311727 \\
\hline 88 & 1100087778366101931 & 213 & 146178119651438213260386312206974243796773058 \\
\hline 89 & 1779979416004714189 & 212 & 90343046356137747723758225621187571439538669 \\
\hline 90 & 2880067194370816120 & 211 & 55835073295300465536628086585786672357234389 \\
\hline 91 & 4660046610375530309 & 210 & 34507973060837282187130139035400899082304280 \\
\hline 92 & 7540113804746346429 & 209 & 21327100234463183349497947550385773274930109 \\
\hline 93 & 12200160415121876738 & 208 & 13180872826374098837632191485015125807374171 \\
\hline 94 & 19740274219868223167 & 207 & 8146227408089084511865756065370647467555938 \\
\hline 95 & 31940434634990099905 & 206 & 5034645418285014325766435419644478339818233 \\
\hline 96 & 51680708854858323072 & 205 & 3111581989804070186099320645726169127737705 \\
\hline 97 & 83621143489848422977 & 204 & 1923063428480944139667114773918309212080528 \\
\hline 98 & 135301852344706746049 & 203 & 1188518561323126046432205871807859915657177 \\
\hline 99 & 218922995834555169026 & 202 & 734544867157818093234908902110449296423351 \\
\hline 100 & 354224848179261915075 & 201 & 453973694165307953197296969697410619233826 \\
\hline 101 & 573147844013817084101 & 200 & 280571172992510140037611932413038677189525 \\
\hline 102 & 927372692193078999176 & 199 & 173402521172797813159685037284371942044301 \\
\hline 103 & 1500520536206896083277 & 198 & 107168651819712326877926895128666735145224 \\
\hline 104 & 2427893228399975082453 & 197 & 66233869353085486281758142155705206899077 \\
\hline 105 & 3928413764606871165730 & 196 & 40934782466626840596168752972961528246147 \\
\hline 106 & 6356306993006846248183 & 195 & 25299086886458645685589389182743678652930 \\
\hline 107 & 10284720757613717413913 & 194 & 15635695580168194910579363790217849593217 \\
\hline 108 & 16641027750620563662096 & 193 & 9663391306290450775010025392525829059713 \\
\hline 109 & 26925748508234281076009 & 192 & 5972304273877744135569338397692020533504 \\
\hline 110 & 43566776258854844738105 & 191 & 3691087032412706639440686994833808526209 \\
\hline 111 & 70492524767089125814114 & 190 & 2281217241465037496128651402858212007295 \\
\hline 112 & 114059301025943970552219 & 189 & 1409869790947669143312035591975596518914 \\
\hline 113 & 184551825793033096366333 & 188 & 871347450517368352816615810882615488381 \\
\hline 114 & 298611126818977066918552 & 187 & 538522340430300790495419781092981030533 \\
\hline 115 & 483162952612010163284885 & 186 & 332825110087067562321196029789634457848 \\
\hline 116 & 781774079430987230203437 & 185 & 205697230343233228174223751303346572685 \\
\hline 117 & 1264937032042997393488322 & 184 & 127127879743834334146972278486287885163 \\
\hline 118 & 2046711111473984623691759 & 183 & 78569350599398894027251472817058687522 \\
\hline 119 & 3311648143516982017180081 & 182 & 48558529144435440119720805669229197641 \\
\hline 120 & 5358359254990966640871840 & 181 & 30010821454963453907530667147829489881 \\
\hline 121 & 8670007398507948658051921 & 180 & 18547707689471986212190138521399707760 \\
\hline 122 & 14028366653498915298923761 & 179 & 11463113765491467695340528626429782121 \\
\hline 123 & 22698374052006863956975682 & 178 & 7084593923980518516849609894969925639 \\
\hline 124 & 36726740705505779255899443 & 177 & 4378519841510949178490918731459856482 \\
\hline 125 & 59425114757512643212875125 & 176 & 2706074082469569338358691163510069157 \\
\hline 126 & 96151855463018422468774568 & 175 & 1672445759041379840132227567949787325 \\
\hline 127 & 155576970220531065681649693 & 174 & 1033628323428189498226463595560281832 \\
\hline 128 & 251728825683549488150424261 & 173 & 638817435613190341905763972389505493 \\
\hline 129 & 407305795904080553832073954 & 172 & 394810887814999156320699623170776339 \\
\hline
\end{tabular}


Table A1. Cont.

\begin{tabular}{cccc}
\hline $\boldsymbol{n}$ & $\boldsymbol{F}_{\boldsymbol{n}}$ & $\boldsymbol{n}$ & $\boldsymbol{F}_{\boldsymbol{n}}$ \\
\hline 130 & 659034621587630041982498215 & 171 & 244006547798191185585064349218729154 \\
131 & 1066340417491710595814572169 & 170 & 150804340016807970735635273952047185 \\
132 & 1725375039079340637797070384 & 169 & 93202207781383214849429075266681969 \\
133 & 2791715456571051233611642553 & 168 & 57602132235424755886206198685365216 \\
134 & 4517090495650391871408712937 & 167 & 35600075545958458963222876581316753 \\
135 & 7308805952221443105020355490 & 166 & 22002056689466296922983322104048463 \\
136 & 11825896447871834976429068427 & 165 & 13598018856492162040239554477268290 \\
137 & 19134702400093278081449423917 & 164 & 8404037832974134882743767626780173 \\
138 & 30960598847965113057878492344 & 163 & 5193981023518027157495786850488117 \\
139 & 50095301248058391139327916261 & 162 & 3210056809456107725247980776292056 \\
140 & 81055900096023504197206408605 & 161 & 1983924214061919432247806074196061 \\
141 & 131151201344081895336534324866 & 160 & 1226132595394188293000174702095995 \\
142 & 212207101440105399533740733471 & 159 & 757791618667731139247631372100066 \\
143 & 343358302784187294870275058337 & 158 & 468340976726457153752543329995929 \\
144 & 555565404224292694404015791808 & 157 & 289450641941273985495088042104137 \\
145 & 898923707008479989274290850145 & 156 & 178890334785183168257455287891792 \\
146 & 1454489111232772683678306641953 & 155 & 110560307156090817237632754212345 \\
147 & 2353412818241252672952597492098 & 154 & 68330027629092351019822533679447 \\
148 & 3807901929474025356630904134051 & 153 & 42230279526998466217810220532898 \\
149 & 6161314747715278029583501626149 & 152 & 26099748102093884802012313146549 \\
150 & 9969216677189303386214405760200 & 151 & 16130531424904581415797907386349 \\
\hline & & & \\
\hline
\end{tabular}

\section{References}

1. Bugeaud, Y.; Mignotte, M.; Siksek, S. Classical and modular approaches to exponential Diophantine equations I. Fibonacci and Lucas powers. Ann. Math. 2006, 163, 969-1018. [CrossRef]

2. Marques, D.; Togbé, A. Perfect powers among Fibonomial coefficients. C. R. Acad. Sci. Paris 2010, 348, 717-720.

3. Luca, F. Fibonacci and Lucas numbers with only one distinct digit. Port. Math. 2000, 57, 243-254.

4. Adegbindin, C.; Luca, F.; Togbé, A. Lucas numbers as sums of two repdigits. Lith. Math. J. 2019, 59, $295-304$. [CrossRef]

5. Luca, F. Repdigits as sums of three Fibonacci numbers. Math. Commun. 2012, 17, 1-11.

6. Marques, D.; Togbe, A. Fibonacci and Lucas numbers of the form $2^{a}+3^{b}+5^{c}$. Proc. Jpn. Acad. Ser. A Math. Sci. 2013, 89, 47-50. [CrossRef]

7. Qu, Y.; Zeng, J.; Cao, Y. Fibonacci and Lucas Numbers of the Form $2^{a}+3^{b}+5^{c}+7^{d}$. Symmetry 2018, 10, 509. [CrossRef]

8. Erduvan, F.; Keskin, R. Fibonacci and Lucas numbers as products of two repdigits. Turk. J. Math. 2019, 43, 2142-2153. [CrossRef]

9. Alvarado, S. D.; Luca, F. Fibonacci numbers which are sums of two repdigits. In Proceedings of the XIVth International Conference on Fibonacci Numbers and Their Applications, Morelia, Mexico, 1-7 July 2011; pp. 97-108.

10. Siar, Z.; Erduvan, F.; Keskin, R. Repdigits as product of two Pell or Pell-Lucas numbers. Acta Math. Univ. Comenian. 2019, 88, 247-256.

11. Ddamulira, M. Repdigits as sums of three Padovan number. Boletín De La Soc. Matemática Mex. 2020, 26, 1-15. [CrossRef]

12. Alahmadi, A.; Altassan, A.; Luca, F.; Shoaib, H. Fibonacci numbers which are concatenations of two repdigits. Quaest. Math. 2020, 43, 1-10. [CrossRef]

13. Trojovský, P. On Terms of Generalized Fibonacci Sequences which are Powers of their Indexes. Mathematics 2019, 7, 700. [CrossRef]

14. Dujella, A.; Pethö, A. A generalization of a theorem of Baker and Davenport. Quart. J. Math. Oxf. Ser. 1998, 49, 291-306. [CrossRef]

(C) 2020 by the author. Licensee MDPI, Basel, Switzerland. This article is an open access article distributed under the terms and conditions of the Creative Commons Attribution (CC BY) license (http:// creativecommons.org/licenses/by/4.0/). 